\title{
Behçet's Disease Presenting with Recurrent Vulvar Abscesses, Oral Ulcers, and Skin Pustules in the Philippines
}

\author{
Ramon B. Larrazabal Jr., MD, ${ }^{1}$ Harold Henrison C. Chiu, $\mathrm{MD}^{2}$ and Cherie Ocampo-Cervantes, $\mathrm{MD}^{3}$ \\ ${ }^{1}$ Division of Medical Oncology, Department of Medicine, Philippine General Hospital, University of the Philippines Manila \\ ${ }^{2}$ Division of Endocrinology, Diabetes, and Metabolism, Department of Medicine, Philippine General Hospital, University of the Philippines Manila \\ ${ }^{3}$ Division of Allergy and Immunology, Department of Medicine, Philippine General Hospital, University of the Philippines Manila
}

\begin{abstract}
Behçet's disease (BD) is a systemic vasculitis characterized by recurrent acute vascular inflammation. There is currently limited epidemiological data in Southeast Asia. We discuss a 26-year-old woman who came to our institution for recurrent vulvar abscesses for a year. Physical examination showed labial, tongue, and vulvar ulcers. Pathergy test was negative and biopsy done were unremarkable. We diagnosed her clinically as a case of Behçet's disease. We started her on colchicine $0.6 \mathrm{mg}$ once daily and educated her on skin, oral, and perineal hygiene. On follow-up after one month, she noted resolution of her lesions. However, symptoms recurred in the last three months after discontinuing treatment. We used telemedicine to communicate due to the COVID-19 pandemic and lockdown, advising her to continue her medication. Timely diagnosis is essential in order to provide symptomatic relief and enhance patient education.
\end{abstract}

Keywords: Behçet's syndrome, ulcer, vasculitis

\section{INTRODUCTION}

Behçet's disease (BD) is a systemic vasculitis with an unknown etiology that affects small, medium, and large blood vessels. It is characterized by recurrent acute inflammation with unpredictable duration and frequency of attacks. It can affect any part of the body with blood vessels. ${ }^{1}$ There is currently scarce data as to the epidemiology of $\mathrm{BD}$ in Southeast Asia. It is rare and probably underreported in the Philippines. ${ }^{2}$ There is a close correlation between the presence of the human leukocyte antigen (HLA) B51 and the prevalence of $\mathrm{BD} .^{3} \mathrm{It}$ is postulated that $\mathrm{BD}$ affects men more than women at $1.5-5: 1$; it has a more serious prognosis in men. ${ }^{1}$

$\mathrm{BD}$ has a peak onset between the age of 20-30 years. The younger the age of onset of symptoms, the more severe and extensive the disease. Women are more likely to manifest with genital ulcers, joint involvement, and erythema nodosum. ${ }^{4}$ Although a positive skin pathergy test is also used

Paper presented in the $22^{\text {nd }}$ Asia-Pacific League of Associations for Rheumatology Virtual Congress on October 24-29, 2020.

Corresponding author: Ramon B. Larrazabal Jr., MD Division of Medical Oncology

Department of Medicine

Philippine General Hospital

University of the Philippines Manila

Taft Avenue, Ermita, Manila 1000, Philippines

Email: ramonlarrazabaljr@gmail.com in diagnosing $\mathrm{BD}$, studies have shown a continual decline in test positivity. ${ }^{5}$ To our knowledge, this is possibly the first reported presentation (vulvar abscesses) of BD in the country.

\section{CASE REPORT}

A 26-year-old woman with an unremarkable past medical and sexual history sought consult at the outpatient 

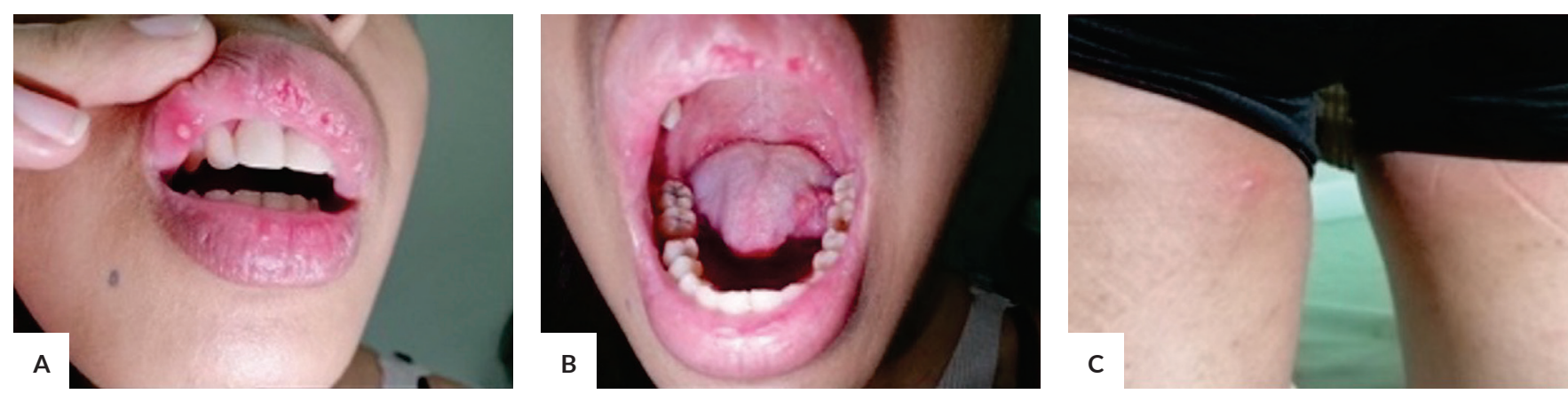

Figure 1. (A) Ulcer at the superolateral labial area with multiple healed areas at the superomedial labial area; (B) Ulcer at the inferolateral border of the left hemitongue; and (C) pustule at the left posterior thigh and multiple indurated and erythematous areas, bilateral posterior thighs.
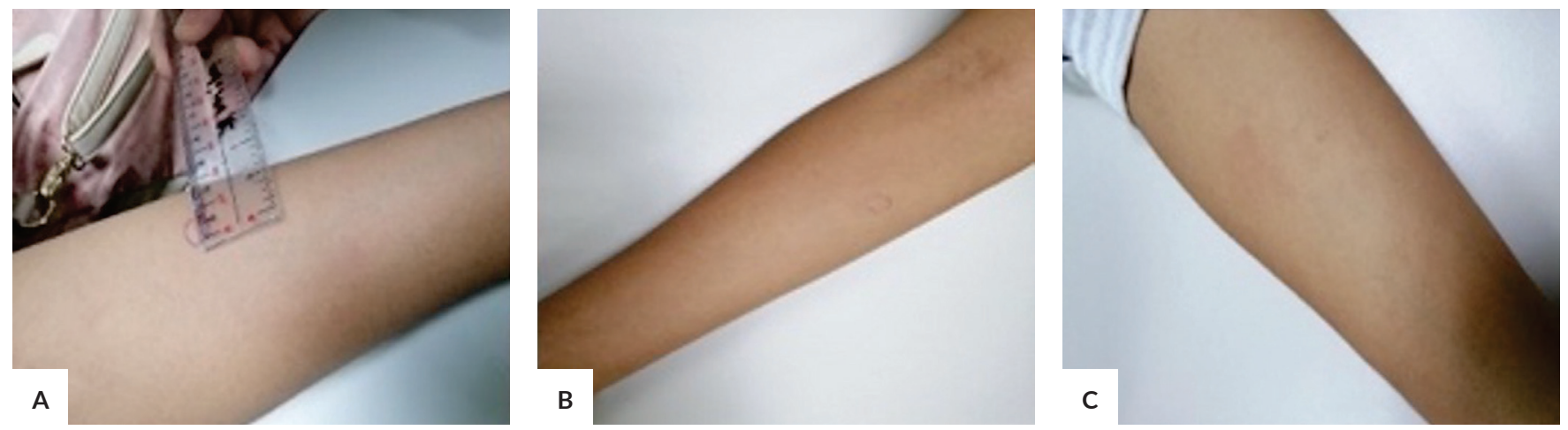

Figure 2. Pathergy test at (A) Day 0, (B) Day 1, and (C) Day 2.

department for oral ulcers, widespread skin pustules, and recurrent vulvar abscesses, including a vulvar abscess that have been present for 12 months. She has no Middle Eastern ancestry and claims to have only Filipino lineage in both sides of the family, with no history of autoimmune disease. Pertinent physical examination findings in oral mucosa are a $0.3 \times 0.3 \mathrm{~cm}$ ulcer at the superolateral labial area, multiple healed areas at the superomedial labial area, and $2.0 \times 1.5 \mathrm{~cm}$ ulcer at the inferolateral border of the left hemitongue. Genitourinary examination revealed a $3.0 \mathrm{x}$ $2.0 \mathrm{~cm}$ ulcer at the inferolateral border of the left vulva. A $0.3 \times 0.3 \mathrm{~cm}$ pustule at the left posterior thigh was noted (Figure 1). There were no vascular bruits and all pulses were full. The rest of the physical examination, including the ophthalmologic examination, was unremarkable.

The patient had anemia with hemoglobin level at $108 \mathrm{~g} / \mathrm{L}$ (Normal value: 120-150 g/L). Blood chemistry, urinalysis, HIV 1 and 2 ELISA tests, aspirate culture, and RPR test were all negative. With a consideration of Behçet's disease, we did a pathergy test (Figure 2) which was negative after 48 hours. A pathergy test was done by an intradermal puncture using a gauge 20 needle at a 45 -degree angle on the flexor region of the forearm. We then waited for 48 hours to see if there was a reaction in the form of a pustule/papule of at least 2 millimeter in diameter. The biopsy of the vulvar lesion revealed chronic active inflammation with vascular endothelial proliferation; a leukocytoclastic vasculitis was observed which is characteristic for patients with $\mathrm{BD}$ (Figure 3). The patient was diagnosed after she fulfilled the International Criteria for Behçet's Disease (ICBD).

She was started on colchicine $0.6 \mathrm{mg}$ once daily and educated on skin, oral, and perineal hygiene. On follow up after one month, her vulvar abscesses, oral ulcers, and skin pustules resolved. However, after three months, there was recurrence of the vulvar abscess. We used telemedicine to communicate with her since our outpatient clinics were closed due to the Coronavirus Disease 2019 pandemic and lockdown. Upon investigation, we found out that she discontinued the colchicine. We advised her to resume her medications. After five months of compliant intake of colchicine, she has reported to be well without any recurrence of her ulcers and abscesses. The investigators obtained an informed consent from the patient to write her case.

\section{DISCUSSION}

BD was historically seen among those who resided in the Silk Road which joined the East and West. However, today it is seen in countries where it was previously extremely rare. This may be because of globalization with increasing 
Behçet's Disease presenting with Recurrent Cutaneous Lesions

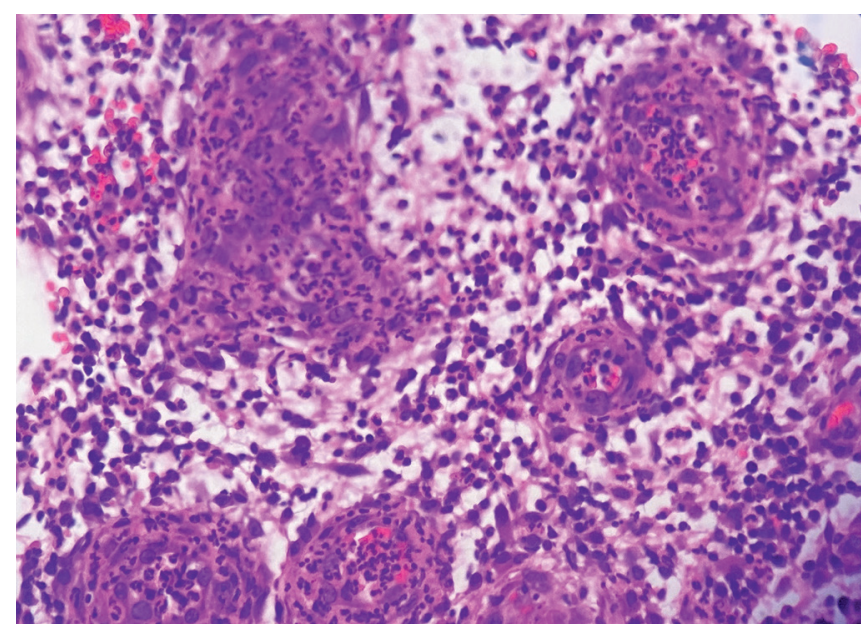

Figure 3. Biopsy of the vulvar lesion in Hematoxylin and Eosin (HPF 400x) staining revealing the characteristic leukocytoclastic vasculitis. The dark violet, multilobed cells (neutrophils) are abundant within the cervical stroma with capillaries exhibiting thickened endothelium which demonstrates reactive atypia.

migration of people from areas where $\mathrm{BD}$ is common to the rest of the world. ${ }^{5}$

The pathogenesis of BD is complex and multifactorial. It has been postulated to be an interaction of innate and adaptive immunity. BD affects almost every part of the body that is vascularized. One of the common manifestations are mucocutaneous. ${ }^{6}$ Among the mucocutaneous manifestations, oral ulcers are the most common. They are present in up to $80 \%$ of the cases and are usually the initial manifestation. These ulcers are painful, well-defined, erythematous, and halo-shaped, often with yellowish pseudo-membranes. They are located in the non-keratinized areas of the oral mucosa. Clinically, the features are almost indistinguishable from recurrent oral aphthosis. ${ }^{7}$ However, the ulcers in $\mathrm{BD}$ are characteristically more than six in number, synchronous occurrence of more than one clinical variant, soft palate and oropharyngeal involvement, and a diffuse enanthem. ${ }^{8}$ Another common manifestation is the presence of genital ulcers that are similar in appearance and clinical course to oral ulcers. The difference is that they are less recurrent, lead to scar formation, and some exhibit necrotic borders. ${ }^{9}$ The most affected site is the scrotum in men and the labia major 1 in women. ${ }^{8}$

A pathergy test elicits a nonspecific hyperreactive lesion in patients with $\mathrm{BD}$, and has been used since 1937 . The test is done by inducing a reaction through trauma to the skin, either intradermal, intravenous, or subcutaneous. A positive reaction is the presence of a lesion in the form of a papule or pustule after 48 hours. Currently, there is no standardized method in doing a pathergy test. ${ }^{10}$

The diagnosis of $\mathrm{BD}$ is clinical and based on the exclusion of other diseases (such as Takayasu's arteritis, giant cell arteritis). The ICBD criteria includes a wide variety of symptoms such as vascular manifestations, skin lesions and neurological manifestations. The criteria permit the early diagnosis of special cases as well as has potential use as a mass screening tool for early referral to expert centers. The main advantage of this criteria is that it can be used with or without the pathergy testing, since it is not routinely performed. The diagnosis of $\mathrm{BD}$ is made when the patient has a score of more than or equal to 4 based on the following: ocular lesions (2 points), genital aphthosis (2 points), oral aphthosis (2 points), skin lesions (1 point), neurological manifestations (1 point), vascular manifestations (1 point), and a positive pathergy test (1 point), which is optional and not part of the primary scoring system. ${ }^{11}$ Our patient had a score of 5 points, which points to a diagnosis of $\mathrm{BD}$ with the exclusion of other diagnoses.

The aim in the management of $\mathrm{BD}$ is disease remission, improved quality of life and prevention of irreversible damage and exacerbation. ${ }^{12}$ This is achieved by addressing inflammation with the use of immunosuppressive agents in manifestations that are mucocutaneous and articular. In patients who have acute exacerbations of mucocutaneous involvement, topical steroids may help in rapid healing. However, for patients who continue to present with such lesions despite the use of colchicine, immunomodulatory or immunosuppressive drugs (such as azathioprine, thalidomide, interferon-alpha, tumor necrosis factors alpha inhibitors or apremilast) can be used. The choice of immunomodulatory or immunosuppressive drug in these patients would depend on individual patient characteristics, safety, cost, availability, and patient preference. ${ }^{13}$ Since $\mathrm{BD}$ has a heterogenous course and presentation, treatment is tailored according to the severity, age at onset, disease duration, organ affected, and frequency of recurrences. ${ }^{3}$ The treatment of $\mathrm{BD}$ is still based mostly on isolated case reports or case series. There are only a few randomized trials on BD. ${ }^{12}$

The goals for the management of oral ulcers in $\mathrm{BD}$ are pain relief and control of inflammation (i.e., decreased recurrences and duration). Limited trials compared the use of placebo with colchicine while others used acyclovir, thalidomide, reamipid, corticosteroids, interferon alpha, and etanercept. Unfortunately, results are inconclusive, and does not support recommending any of the drugs as the gold standard for the treatment of oral ulcers in $\mathrm{BD} .{ }^{14}$

Common clinical practices in the management of $\mathrm{BD}$ are education in oral hygiene and food intake (i.e. avoid salty or spicy food), mouthwashes (i.e. chlorhexidine, triclosan, etc.), lasers, silver nitrate, or topical corticosteroids. When systemic treatment is highly suggested; colchicine, for its inhibition of neutrophil chemotaxis, is initiated alone or with an oral corticosteroid. For this patient, we educated her on proper hygiene not only in the oral aspect but also perineal and the general skin. We also started her on colchicine $0.6 \mathrm{mg}$ once daily. She reported resolution of the oral ulcers and skin lesions. 


\section{CONCLUSION}

In summary, this is the case of a 26-year-old Filipino woman who presented with recurrent cutaneous lesions and was diagnosed with Behçet's disease using the criteria of the ICBD. An extensive diagnostic work-up ruled out immunodeficiency, malignancy, and infection as causes for her presentation. We gave her colchicine to control the systemic inflammation, which led to resolution of her symptoms. However, due to problems in logistics brought about by the pandemic, follow up was disrupted. We used telemedicine to continue her care despite the COVID-19 pandemic and lockdown in the country.

\section{Acknowledgment}

The authors would like to express their gratitude to the Division of Allergy and Immunology for comanaging the patient.

\section{Statement of Authorship}

$\mathrm{RBL}$ and $\mathrm{HHC}$ were involved in the care of the patient and in writing this case report. COC was the consultant who checked our work and oversaw our management of the patient.

All authors participated in the data collection and analysis and approved the final version submitted.

\section{Author Declaration}

All the authors declared no conflicts of interest.

\section{Funding Source}

This study was self-funded.

\section{REFERENCES}

1. Scherrer MAR, Rocha VB, Garcia LC. Behçet's disease: review with emphasis on dermatological aspects. An Bras Dermatol. 2017; 92(4):452-64.

2. Limgenco-Hipe JR, Salido E, Penserga E. Disease characteristics of Behçet's disease among Filipino patients seen in rheumatology clinics. Philipp J Intern Med. 2017; 55(4):1-5.

3. Alpsoy E. Behçet's disease: a comprehensive review with a focus on epidemiology, etiology and clinical features, and management of mucocutaneous lesions. J Dermatol. 2016; 43:620-32.

4. Hatemi G, Seyahi E, Fresko I, Talarico R, Hamuryudan V. Behçet's syndrome: a critical digest of the 2014-2015 literature. Clin Exp Rheumatol. 2015; 33:S3-14.

5. Davatchi F, Chams-Davatchi C, Ghodsi Z, Shahram F, Nadji A, Shams H, et al. Diagnostic value of pathergy test in Behcet's disease according to the change of incidence over the time. Clin Rheumatol. 2011; 30:1151-5.

6. Zeidan MJ, Saadoun D, Garrido M, Klatzmann D, Six A, Cacoub P. Behçet's disease physiopathology: a contemporary review. Auto Immun Highlights. 2016; 7:4-4.

7. Baş Y, Seçkin HY, Kalkan G, Takcı Z, Önder Y, Çıtıl R, et al. Investigation of Behçet's disease and recurrent aphthous stomatitis frequency: the highest prevalence in Turkey. Balkan Med J. 2016; 33:390-5

8. Alpsoy E, Zouboulis CC, Ehrlich GE. Mucocutaneous lesions of Behcet's disease. Yonsei Med J. 2007; 48:573-85.

9. Lee S, Bang D, Lee E, Sohn S. Behçet's Disease: a guide to its clinical understanding. New York: Springer-Velag; 2001.

10. Kutlubay Z, Tuzun Y, Wolf R. The pathergy test as a diagnostic tool. Skinmed. 2017: 15(2):97-104.

11. International Team for the Revision of the International Criteria for Behçet's Disease (ITR-ICBD). The International Criteria for Behçet's Disease (ICBD): a collaborative study of 27 countries on the sensitivity and specificity of the new criteria. J. Eur. Acad. Dermatol. Venereol. 2014 Mar; 28(3):338-47.

12. Saleh Z, Arayssi T. Update on the therapy of Behçet's disease. Ther Adv Chronic Dis. 2014; 5:112-34.

13. Hatemi G, Silman A, Bang D, Bodaghi B, Chamberlain AM, Gul A, et al. Management of Behçet's disease: a systematic literature review for the European League Against Rheumatism evidence-based recommendations for the management of Behçet's disease. Ann Rheum Dis. 2009; 68:1528-34.

14. Taylor J, Glenny AM, Walsh T, Brocklehurst P, Riley P, Gorodkin $\mathrm{R}$, et al. Interventions for the management of oral ulcers in Behçet's disease. Cochrane Database Syst Rev. 2014; 25:CD011018 